\title{
Comment on: Approaching Chronic cough
}

Singapore Med J 2017; 58(9): 573 doi: 10.11622/smedj.2017090

Dear Sir,

I found the Singapore Medical Journal article about approaching chronic cough very interesting. ${ }^{(1)}$ It focused on common causes of chronic cough (cough with duration $\geq 3$ weeks) such as post-infectious cough, cough variant asthma, postnasal drip syndrome, gastro-oesophageal reflux disease, smoker's cough, use of angiotensin-converting enzyme inhibitors, and nonasthmatic eosinophilic bronchitis. Besides the causes listed, endobronchial tuberculosis (EBTB), which is tuberculous infection of the tracheobronchial tree with microbial and histopathological evidence, should be mentioned as one of the aetiologies of chronic cough.

The reasons are as follows. First, prolonged cough often manifests as the primary symptom in patients with EBTB, and about $10 \%-20 \%$ of these patients show a normal chest radiograph. ${ }^{(2)}$ For instance, Lee et al described a case of EBTB with similar features in 2004. ${ }^{(3)}$ Diagnosing EBTB requires a high index of suspicion and for bronchoscopy to be performed appropriately. Second, the annual incidence of new pulmonary tuberculosis cases in Singapore has been increasing in recent years, according to research by Chee et al in 2012.(4) This change will obviously affect the incidence rate of EBTB. Although screening for pulmonary tuberculosis in patients with prolonged cough is critical, the sputum smear to test for acid-fast bacillus has shown low sensitivity in detecting EBTB. ${ }^{(2)}$ Hence, awareness of EBTB among patients with prolonged cough has an important diagnostic role.

In conclusion, although EBTB is uncommon, it should be considered as one of the causes of chronic cough, especially in countries with an increasing incidence of pulmonary tuberculosis.

Yours sincerely,

Ho Lam Nguyen

Department of Internal Medicine, University of Medicine and Pharmacy, Ho Chi Minh City, Vietnam, bsholam1986@gmail.com

\section{ReFERENCES}

1. Poulose V, Tiew PY, How CH. Approaching chronic cough. Singapore Med I 2016; 57:60-3.

2. Shahzad T, Irfan M. Endobronchial tuberculosis-a review. J Thorac Dis 2016; 8:3797-802.

3. Lee YH, Sin Fai Lam KN. Endobronchial tuberculosis simulating bronchial asthma. Singapore Med J 2004; 45:390-2.

4. Chee CB, Wang YT. TB control in Singapore: where do we go from here? Singapore Med J 2012; 53:236-8. 